\title{
Automated Unauthorized Parking Detector with Smart Vehicle Parking
}

\author{
Sridevi Garikapati and S. Sarath Chandra
}

\author{
Department of Electronics and Communication Engineering, QIS College of Engineering and Technology, \\ Ongole-523272, Andhra Pradesh, India; sreedevi.ece426@gmail.com, ssschandra@gmail.com
}

\begin{abstract}
Background/Objectives: In today's world there is a rapid increment in vehicle movement and to resolve the vehicle parking problem, there is a need to employ technology. Methods/Findings: We introduce a RFID based technique; using this RFID technique we can able to resolve the parking problem without considering the manpower. In this RFID, technique we use LCD display, sensors and GSM Modem. Application: In this approach. SMS service plays a major role. By using RFID tags, we able to identify the vehicle having a slot in parking lot or not.
\end{abstract}

Keywords: Detector, LCD Display, RFID Tags, Unauthorized Parking

\section{Introduction}

Vehicle parking management, like any other field, requires automation to make sure the security and luxury. With the enormous increase in population and an increasing amount of vehicles, it has ended up difficult to find enough car space for parking in public places. Thus, the idea of a computerized vehicle parking device and unauthorized parking detector has been a need for a while. The important motivation is to keep the security of the parking place and reduce the time loss in the car parking zone. The venture aims to make an automatic parking device which facilitates to restrict unauthorized vehicle parking within the restricter area and generates fine if an automobile is parked violating the regulations; it is done by means of sending an SMS to the registered proprietor of the vehicle ${ }^{1}$.

With the registration numbers, the vehicles are identified by the system. Using the system we can able to identify whether the vehicle is authorized or not. If it's authorized vehicle then it will be allotted with parking space. If it is not a authorized vehicle, the system should give five to ten minutes of time to pick or drop of a passenger to depart. Otherwise, its owner may be charged with a pre-allocated fine as overdue price. There will also be a machine of parking fees in an hourly foundation. The sensors within the parking space will show the unoccupied space that can be allotted for parking. It is was hoping that this venture may additionally have an amazing effect in making lives simpler and contribute to building smart towns.

$\mathrm{In}^{2}$ used IR sensor modules for the LED to light up. Wireless sensor era offering superior features like remote parking monitoring, automated guidance, and parking reservation mechanism ${ }^{3}$. There also a system that makes use of automatic variety plate popularity cameras to successfully manipulate, display and defend the parking facilities of a university ${ }^{4}$.

\section{System Architecture}

The gadget includes RFID module, GSM modem, Infrared Sensor module, Servo motor, Arduino UNO, Arduino Mega. The machine architecture is shown in Figure 1. 
RFID: The whole RFID device includes RFID tags, two RFID modules and two RFID Reader every at the entry factor and exit factor. The RFID reader is attached to the RFID module. The RFID tags are read via the RFID reader to determine whether the RFID tag range suits with any of the RFID numbers of the registered motors. The RFID at the access factor facilitates to decide access time for a septic automobile and the only at the exit point is used to decide the parking fee for that automobile depending at the time or going out. The RFID used for entry is connected to Arduino Mega and the one that is used to go out is connected to Arduino UNO.

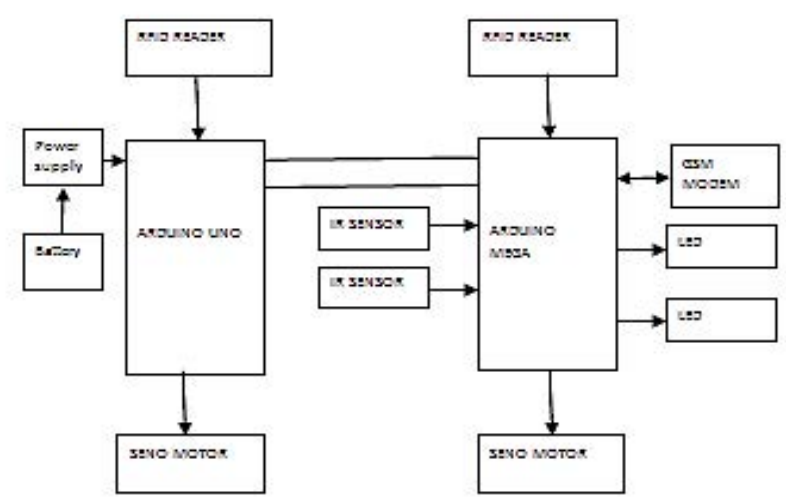

Figure 1. System Architecture Block Diagram.

GSM Modem: The GSM modem is used for the transfer of SMS's for unauthorized parking. It has a SIM card and operates like a cell phone. When a GSM modem is connected to a laptop, this lets in the pc to use the GSM modem to talk over the mobile community.

Servo Motor: The servo motor is used for controlling the parking gate.

Infrared Sensor Module: The IR Sensors helps to detect the presence of the vehicle in the parking lots.

LED: The LED lighting turns on every time a car is detected in the car parking zone. Also, those lights get on every time a registered automobile is identified at the entrance in any other case they stay off.

The common functionality of the gadget, in line with the top view of the version given in Figure 2, is as follows. If any unregistered vehicle enters in to an entry point, the sensor will verify the registered number of the vehicle and the verified data will send to server. If it is a registered vehicle then RFID card will be verified with validity ${ }^{5}$. If the RFID is active mode then the vehicle can move in to check in. when the vehicle is checked in, the time of entry is noted at the server. When the vehicle is away from the parking area. i.e. left the parking slot or going out of the area from the server bill will be generated when we pays the bill then only vehicle can move away from the Check out area.
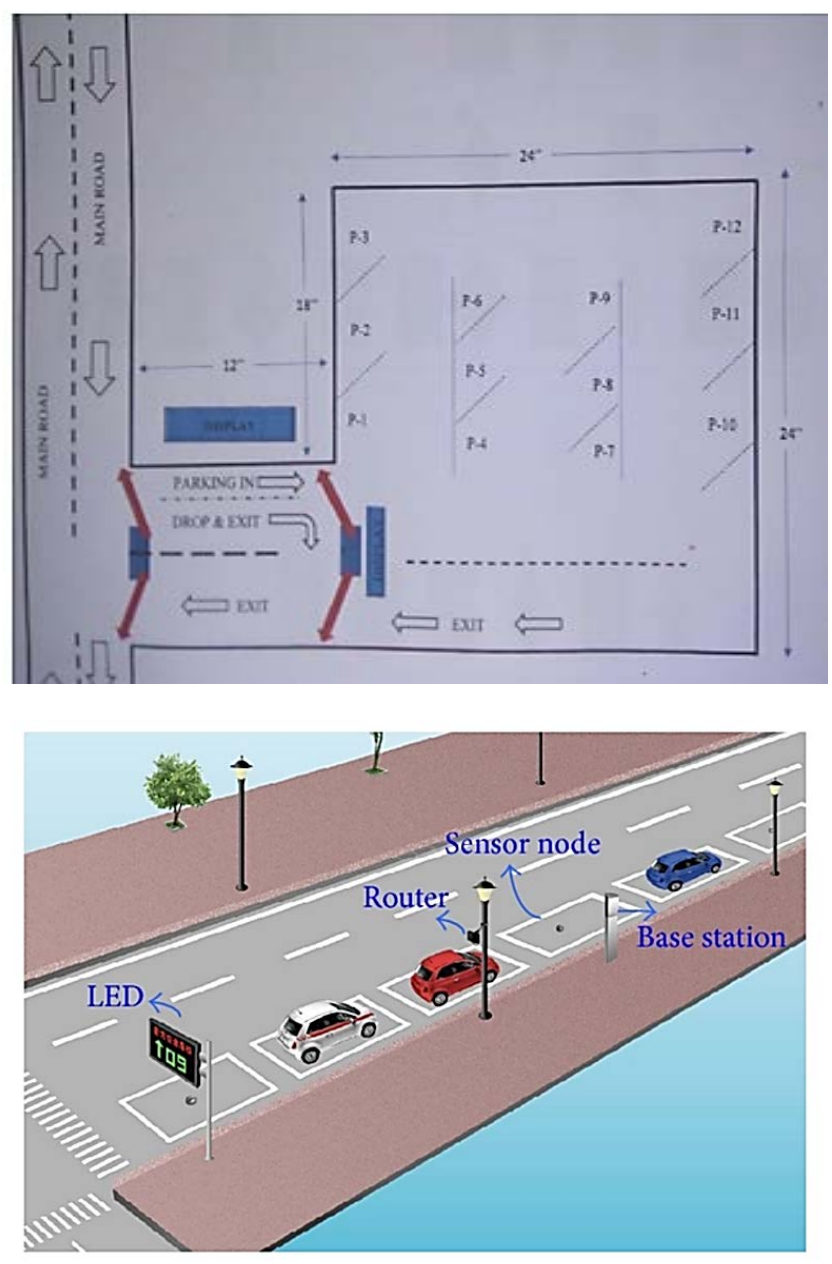

Figure 2. Top View of Proposed Model.

In the first Arduino Uno, Figure 3, the pins $2 \& 3$ are used as receiver and transmitter simultaneously of the RFID reader module. That means the readings obtained from the RFID reader module is taken because the access of a selected vehicle and its analyzing goes to the pin which acts as the receiver and the pin 3 is the transmitter.

This is particularly the main entrance to the automobile parking space. Here 2 servo motors are connected one is connected to the pin 5 which acts as an access barrier which opens if the card punched in common place 
and the alternative servo motor is hooked up to the pin 7 which acts for the go out barrier. The time is calculated and it's time saved in the database. A GSM module for sending SMS in case of unauthorized parking is ready at pins $11 \& 12$ and there may be a buzzer which is ready to switch on the pin 9 of the Arduino.

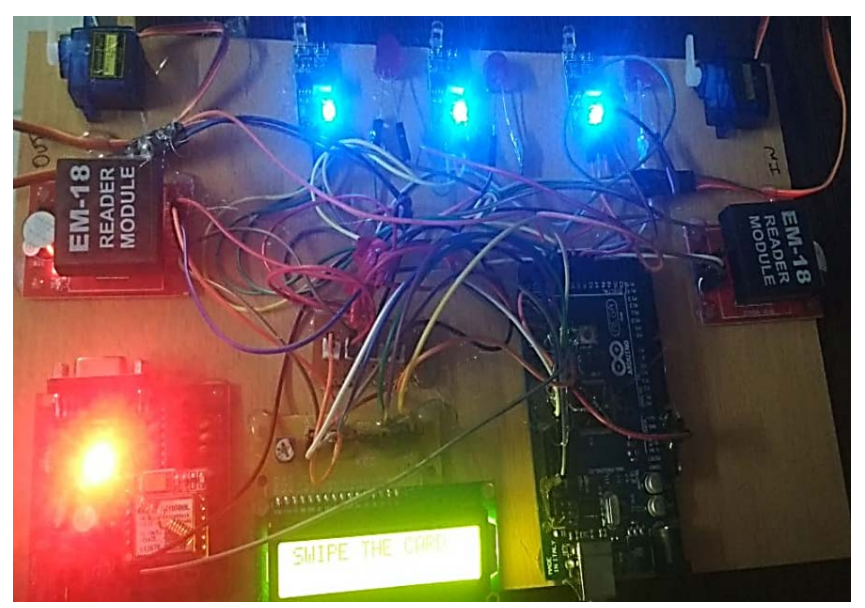

Figure 3. Hardware Model Kit.

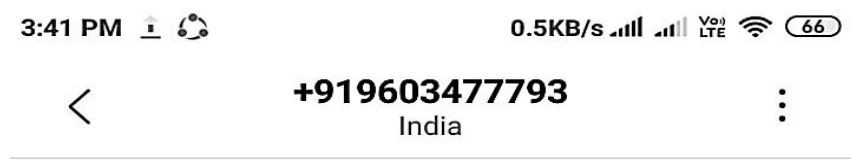

3:40 PM

\section{UnAuthorized Vehicle Card} Detected

\section{Trext message}

2

个

Figure 4. Text Message Alert, When Unauthorized Vehicle Enters in Parking Zone.

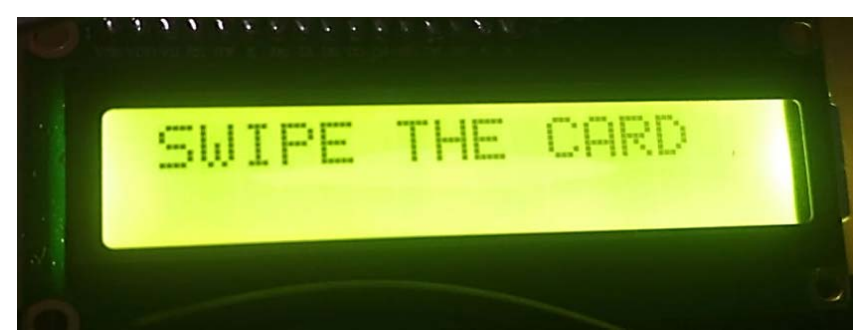

Figure 5. LCD Output Display Shows the Request for Vehicle Card.

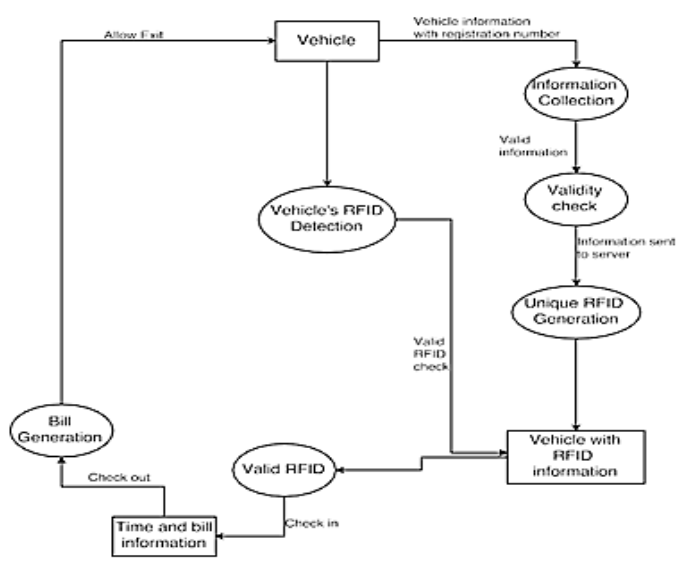

Figure 6. Data Flow of the System.

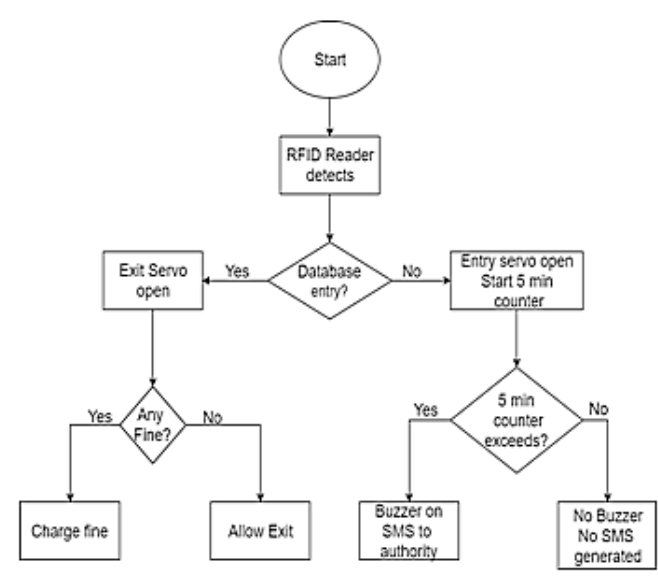

Figure 7. Main Gate Entry.

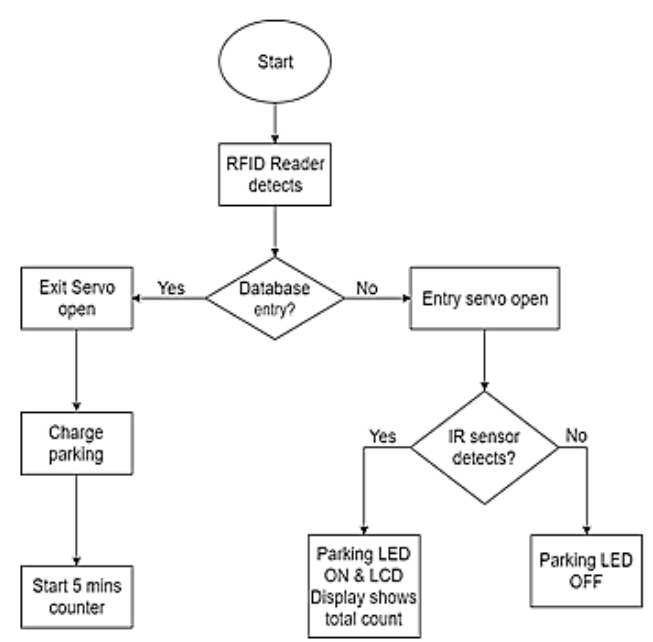

Figure 8. Parking Gate Entry. 
In the second Arduino Uno, the pins 2 and 3 are used as receiver and transmitter simultaneously of the RFID reader module. That way the readings received from the RFID reader module is taken as they go out of a specific vehicle and its reading is going to pin 2 which acts as the receiver and the pin 3 is the transmitter. Just like the preceding Arduino here there are servo motors one connected to the pin five acting as an entry barrier and the opposite at pin 7 appearing because of the go out barrier. Here the time is also taken under consideration and stored in the database ${ }^{6}$.

A third Arduino mega is used for the LCD display, the IR sensors in the parking zone and the LEDs and IR sensors within the specific parking. The IR sensors are connected to the pins A0, A1, A2 \& A3 and their output LEDs are related to $9,8,7$ and 6 respectively. The LCD shows the number of cars currently present in the parking location.

\section{System Implementation}

At first, an automobile needs to be registered to advantage access in the constrained sector and the parking vicinity. So they're first registered through the RFID reader in the primary entry. If a vehicle is to be canceled out of the registration, it is also executed in the fundamental entry RFID reader ${ }^{7}$. Then if the auto is registered, it punches the RFID tag to the main entrance and the gate opens recording the time of the doorway. From then onwards, a time of 5 minutes begins to check whether the vehicle leaves or enters the parking zone. If the vehicle stays in the no parking vicinity, an SMS is dispatched to the proprietor and authority informing about the unlawful act and fine is charged in Figure 4. With the timer crossing its limit, a buzzer begins alarming. If the automobile punches the cardboard for the parking entry, then the timer is stopped and the time of parking front is mentioned. Inside the parking zone (Figure 3), in whichever parking the car park, the corresponding LED is lit and the quantity of cars inside the lot is displayed in the LCD display (Figure 5). When the car punches the RFID reader in the parking access once more, then the time is cited and parking rate in step with the length of parking is charged. And from then onward, some other timer starts to check if the car leaves in the given time, otherwise, the buzzer will cross on, an SMS will be sent and fine will be charged. When the car finally punches the primary entry RFID, then the time is stated and saved into account inside the database and the vehicle leaves the restrained area. These overall functionalities are shown within the Figures 6-8.

\section{Conclusion}

As the variety of motors keeps increasing, parking areas are at a top rate in metropolis streets. Moreover, due to the lack of know-how approximately street parking spaces, circling the blocks searching for an empty spaces and waste of time and oil of the vehicle but also will increase city congestion. In order to build smart town, effective parking system is needed. The system will reduce or manage unauthorized parking but also reduces traffics congestion and saves time (Figure 4). Thus, our device is appropriate to ensure the right parking control machine in any public places.

\section{Future Extension}

In near future, when the technology of automated vehicle parking system will be in use in a larger scale, this system can be updated and made more advanced so that this vehicle parking system becomes convenient to the users. Thus, we have the urge to upgrade our system by ensuring vehicle registration via any smart card, fine and parking charge collection via online payment and parking slot booking in advance via SMS.

\section{References}

1. Gupta A, Kulkarni S, Jathar V, Sharma V, Jain N. Smart Car Parking Management System Using IoT. American Journal of Science, Engineering and Technology. 2017; 2:112-9.

2. Olasupo TO, Otero CE, Otero LD, Olasupo KO, Kostanic I. Path Loss Models for Low-Power, Low-Data-Rate Sensor Nodes for Smart Car Parking Systems. IEEE Transactions on Intelligent Transportation Systems. 2017; 19(6):1774-83. https://doi.org/10.1109/TITS.2017.2741467

3. Gandhi BMK, Rao MK. A prototype for IoT based car parking management system for smart cities. Indian Journal of Science and Technology. 2016; 9(17):1-6. https://doi. org/10.17485/ijst/2016/v9i17/92973

4. Hilman A, Maizate A. A study of self-organization protocols in a wireless sensor network. Mediterranean Telecommunications. 2017; 7(2):1-20.

5. Car park management with networked wireless sensors and active RFID. Available from: https://ieeexplore.ieee.org/ abstract/document/7293372. Date accessed: 21/05/2015. 
6. Wagh SS, More A, Kharote PR. Performance Evaluation of IEEE 802.15.4 Protocol under Coexistence of WiFi 802.11b. Procedia Computer Science. 2015; 57:745-51. https://doi. org/10.1016/j.procs.2015.07.467
7. Patil M, Bhonge VN. Wireless sensor network and RFID for smart parking system. International Journal of Emerging Technology and Advanced Engineering. 2013; 3:188-92. 